\title{
Retranslocación de nutrientes en especies dominantes de bosques del Espinal Mesopotámico (Argentina)
}

\author{
Nutrient resorption in dominant species of forests in the 'Espinal Mesopotámico’ (Argentina) \\ Carlos A Mendoza ${ }^{\text {a*, }}$ M Belén Turrión ${ }^{\mathrm{b}}$, Pablo G Aceñolaza ${ }^{\mathrm{c}}$, Juan F Gallardo ${ }^{\mathrm{y}}$ y Valentín Pando ${ }^{\mathrm{e}}$ \\ *Autor de correspondencia: ${ }^{a}$ FCyT, Universidad Autónoma de Entre Ríos, Empalme Neid, Villaguay 3240, \\ Entre Ríos, Argentina, tel.: +54.3455.423383, tatomen02@hotmail.com \\ b Universidad de Valladolid, Área de Edafología y Química Agrícola e Instituto Universitario de Gestión Forestal Sostenible, \\ E.T.S.II.A.A., Campus Palencia, Palencia 34071, España, Avda. Madrid 57, bturrion@agro.uva.es \\ c CICyTTP-CONICET y FCyT-UADER, Materi y España, Diamante 3105, Entre Ríos, Argentina, acenolaza@gmail.com \\ ${ }^{d}$ C.S.I.C., IRNASa, Salamanca 37071. España, juanf.gallardo@ csic.es \\ e Universidad de Valladolid, Departamento de Estadística e Inv. Operativa, E.T.S.II.A., Campus Avda. Madrid 57, \\ Palencia 34071, España, vpando@eio.uva.es
}

\begin{abstract}
SUMMARY
The resorption of nutrients of dominant species in a successional sequence of forests of the Argentinean Mesopotamian Espinal was studied for two years (2009-2010). The aim was to compare nutrient resorption (nitrogen, phosphorus, potassium, calcium, magnesium and sulphur) among species of the Fabaceae (Acacia caven, Prosopis affinis and Prosopis nigra with the capacity of biologically fixing the atmospheric nitrogen) and Celtidacea families (Celtis tala, without the capacity of biologically fixing the atmospheric nitrogen). Samples of mature leaves (collected in spring) and senescent leaves (collected in autumn) were chemically analyzed to determine nutrient concentration; afterwards, resorption rates were calculated $\left(R c_{1}\right.$ and $\left.R c_{2}\right)$. It was found that the higher nitrogen resorption was associated with the inability of biologically fixing the atmospheric nitrogen, having both indexes a maximum nitrogen resorption in C. tala and a minimum in A. caven. On the contrary, the other elements ( $\mathrm{P}, \mathrm{K}, \mathrm{Ca}, \mathrm{Mg}$ and $\mathrm{S}$ ) showed resorption differences in one of the indexes used, indicating a higher resorption in Fabaceae in contrast with Celtidaceae, associated with a higher foliar demand of these elements.
\end{abstract}

Key words: Fabaceae, Celtidacea, resorption.

\section{RESUMEN}

Se estudió durante dos años (2009 y 2010) la retranslocación de nutrientes en especies dominantes de bosques correspondientes a una secuencia sucesional del Espinal Mesopotámico. El objetivo fue comparar la retranslocación de nutrientes (nitrógeno, fósforo, potasio, calcio, magnesio y azufre) entre especies de familias fabáceas (Acacia caven, Prosopis affinis y Prosopis nigra; con capacidad de fijar biológicamente el nitrógeno atmosférico) y celtidáceas (Celtis tala, sin capacidad de fijar biológicamente el nitrógeno atmosférico). Se analizaron químicamente las muestras de hojas maduras (recolectadas en primavera) y senescentes (recolectadas en otoño) para determinar la concentración de nutrientes y luego se calcularon los índices de retranslocación $\left(R c_{1}\right.$ y $\left.R c_{2}\right)$. Se determinó que la mayor retranslocación del nitrógeno estuvo asociada la imposibilidad de fijar biológicamente el nitrógeno atmosférico, observándose que ambos índices denotaron una máxima retranslocación de nitrógeno en C. tala y mínima en A. caven. Contrariamente, los demás elementos (P, K, Ca, Mg y S) mostraron diferencias en la retranslocación en uno de los índices utilizados, indicando mayor resorción en fabáceas respecto a celtidácea, asociado a una mayor demanda foliar de estos elementos.

Palabras clave: fabáceas, celtidáceas, resorción.

\section{INTRODUCCIÓN}

La retranslocación o resorción es un proceso fisiológico referido a la utilización repetida de las mismas unidades de algún nutriente por parte del árbol; se realiza con la finalidad de maximizar su uso cuando estos son escasos en el suelo (Del Arco et al. 1991) evitando así su pérdida (Vitousek 1982). Se considera a la retranslocación como un mecanismo eficaz para la conservación de nutrientes por aparte del árbol y es característico en un gran número de especies, siendo la retranslocación en árboles de bosques templados poco eficientes en relación a lo observado en bosques tropicales (Vitousek 1982).

De las especies evaluadas en bosques de un área del Espinal Mesopotámico, el 75 \% pertenecen a la familia fabácea (Prosopis nigra (Griseb.) Hieron (algarrobo negro), Prosopis affinis Spreng (ñandubay) y Acacia caven (Molina) Molina (espinillo)) y el $25 \%$ a la familia celtidácea (Celtis tala Gillies ex Planch. (tala)). Las especies mencionadas han sido evaluadas en sus aportes de hojas al 
suelo, observándose diferencias significativas entre ellas (Mendoza et al. 2012).

Es de destacar que no existen antecedentes en estudios de retranslocación en especies representativas del espinal Mesopotámico; como tampoco hay análisis químicos relativos a la concentración de nutrientes (nitrógeno, fósforo, potasio, calcio, magnesio y azufre) en hojas maduras $(\mathrm{Hm})$ o senescentes $(\mathrm{Hs})$ de las mencionadas especies.

Siendo las fabáceas las únicas con capacidad de fijar biológicamente el nitrógeno atmosférico, resulta de interés comparar la retranslocación del nitrógeno y de los demás elementos (P, K, Ca, Mg y S) entre especies de las familias Fabaceae y Celtidaceae.

Como hipótesis de trabajo se plantea que la retranslocación del nitrógeno entre las familias fabáceas y celtidáceas será diferente en función de la posibilidad de fijar biológicamente el nitrógeno atmosférico; siendo la retranslocación de los demás elementos (P, K, Ca, Mg y S) una expresión de la maximización de su uso.

Por tanto, el objetivo general del trabajo es conocer si existen diferencias entre la retranslocación entre especies de las familias Fabaceae y Celtidaceae.

Los objetivos específicos de este estudio son:

- Comparar la concentración en elementos químicos ( $\mathrm{N}$ y $\mathrm{P}, \mathrm{K}, \mathrm{Ca}, \mathrm{Mg}$ y S) de las hojas maduras y hojas senescentes en especies de las familias Fabaceae y Celtidaceae.

- Comparar la retranslocación entre nitrógeno y fósforo, potasio, calcio, magnesio y azufre en especies de las familias Fabaceae y Celtidaceae, utilizando dos índices diferentes.

\section{MÉTODOS}

Descripción del área de estudio. El Espinal Mesopotámico es un ecosistema boscoso de especies arbóreas xerófilas, arbustivas y herbáceas temporales; su estrato herbáceo es de gran valor forrajero, por lo que se utiliza como pastizal para el ganado vacuno en Argentina. Esta formación boscosa se ubica entre los $28^{\circ}$ y los $40^{\circ}$ de latitud Sur y se extiende como un gran arco por el Oeste, rodeando el pastizal pampeano y ubicándose al Sur del Parque Chaqueño y comprende los bosques de la provincia fitogeográfica del espinal, distrito del Ñandubay (Lewis et al. 2004). En muchas áreas de la provincia de Entre Ríos (Argentina) se observa una intensa modificación del Espinal Mesopotámico en el paisaje rural debido al avance de la frontera agrícola (Muñoz et al. 2005) con un patrón de heterogeneidad creciente. Como consecuencia de ello, los bosques prístinos fueron transformados en una "secuencia sucesional" caracterizada por bosques degradados o secundarios debido a la extracción de árboles con finalidad maderera, centrado en la corta de Prosopis nigra o para uso de combustible como en los casos de Prosopis affinis, Acacia caven y Celtis tala.

El estudio se realizó en el departamento de Villaguay (Entre Ríos, Argentina) y comprende un área de unos $1.600 \mathrm{~km}^{2}$ (figura 1).

Su clima se clasificó como templado-húmedo. Las precipitaciones medias anuales en el área de estudio fueron de $1.000 \mathrm{~mm}_{\text {año }}{ }^{-1}$ con importante variabilidad interanual (INTA 2000); las lluvias se distribuyen a lo largo del año, pero con notables incrementos en otoño y primavera (figura 2).

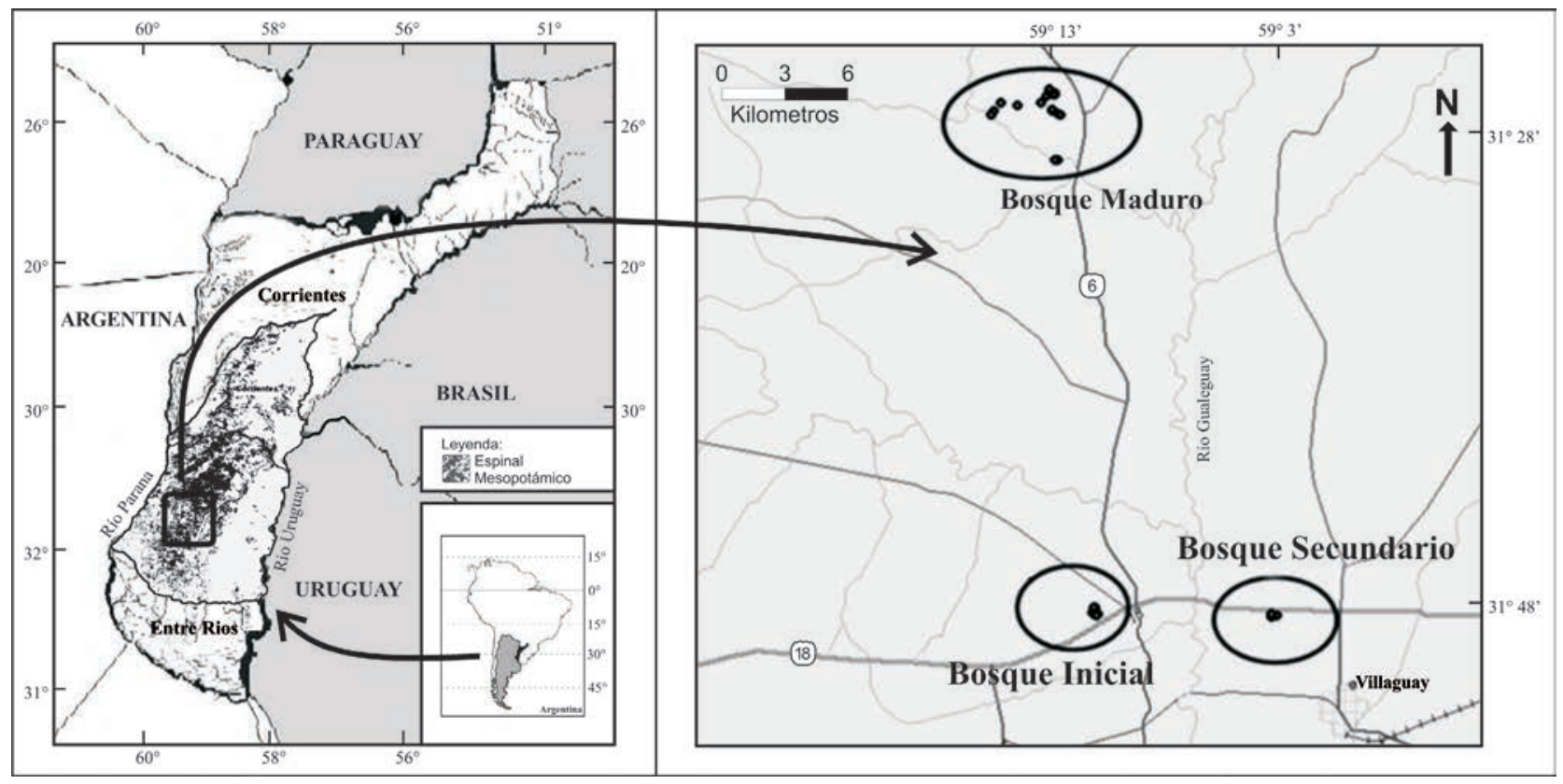

Figura 1. Ubicación geográfica del área de estudio, delimitándose los bosques seleccionados. Bosque inicial dominado por $A$. caven. Bosque secundario dominado por $P$. affinis. Bosque maduro dominado por P. nigra y C. tala.

Geographical location of the study area, indicating selected forests. Initial forest dominated by A. caven. Secondary forest dominated by P. affinis. Mature forest dominated by P. nigra and C. tala. 
La temperatura media anual fue de $16^{\circ} \mathrm{C}$, siendo la media del mes más frío $11^{\circ} \mathrm{C}$ (julio) y del mes más cálido $25^{\circ} \mathrm{C}$ (febrero, figura 2). Se pasa de una estación a la otra de forma gradual, aunque es común tener en verano días con temperaturas mínimas inferiores a $10^{\circ} \mathrm{C}$ y en invierno días con máximas superiores a los $30^{\circ} \mathrm{C}$.

Desde el punto de vista geológico durante el Cuaternario se depositaron los principales sedimentos de la formación 'Hernandarias' (Pleistoceno medio) que conformaron los materiales parentales de los suelos del área de estudio (de origen lacustre-palustre). Dichos materiales dieron origen a suelos de textura arcillosa, con altos contenidos de arcilla (montmorillonita), profundos, con escaso drenaje, con concresiones calcáreas a partir de los $90 \mathrm{~cm}$ y deficitarios en fósforo asimilable en sistemas agrícolas (INTA 2000). Taxonómicamente los suelos del área de estudio se corresponden al orden Vertisol, más concretamente Peludert árgico (INTA 2000). Las características edáficas relacionadas a las propiedades de los Vertisoles de la secuencia sucesional estudiada se describen en el cuadro 1, destacándose que mientras los valores de carbono orgánico, nitrógeno total, fósforo asimilable y potasio asimilable aumentan a medida que la sucesión forestal avanza; la relación edáfica carbono:nitrógeno varió en torno de 12 (cuadro 1).

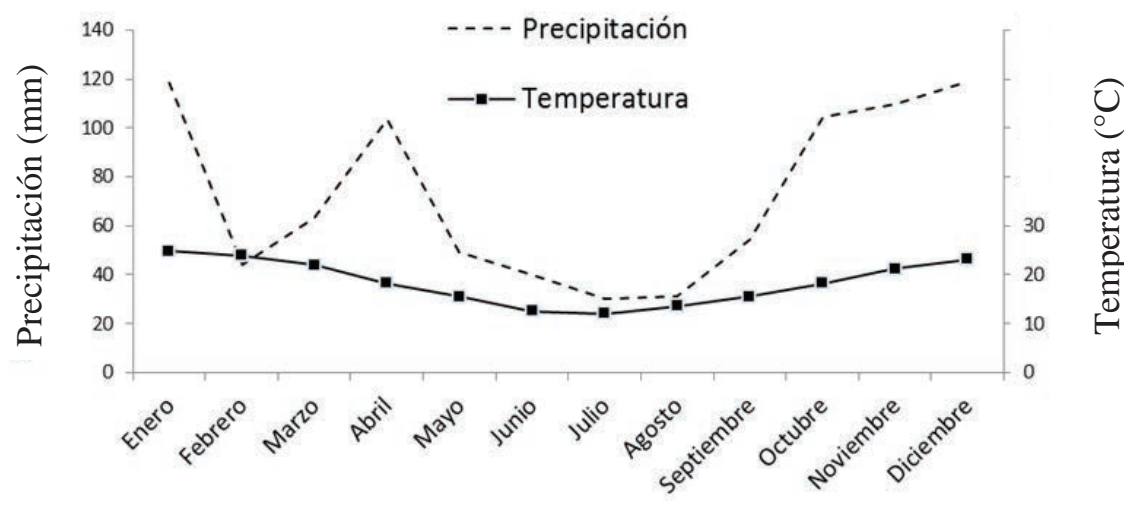

Figura 2. Diagrama ombrotérmico correspondiente al clima de la zona de estudio (departamento Villaguay, Entre Ríos, Argentina). Temperature and rainfall diagram corresponding to the study area (Villaguay, Entre Ríos, Argentina).

Cuadro 1. Características generales de las tres parcelas correspondientes a la secuencia sucesional en bosques del Espinal Mesopotámico (Centro-norte de Entre Ríos, Argentina).

General characteristics of the three plots corresponding to the successional sequence in forests of the Mesopotamian Espinal (Centre-North of Entre Ríos, Argentina).

\begin{tabular}{|c|c|c|c|}
\hline Parcelas & Bosque inicial & Bosque secundario & Bosque maduro \\
\hline Latitud & $31^{\circ} 48^{\prime} 26^{\prime \prime} \mathrm{S}$ & $31^{\circ} 47^{\prime} 46^{\prime \prime} \mathrm{S}$ & $31^{\circ} 28^{\prime} 11^{\prime \prime} \mathrm{N}$ \\
\hline Longitud & $59^{\circ} 11^{\prime} 23^{\prime \prime} \mathrm{O}$ & $59^{\circ} 4$ ’14”O & $59^{\circ} 14^{\prime} 20^{\prime \prime} \mathrm{O}$ \\
\hline Orden & Vertisol & Vertisol & Vertisol \\
\hline Arena (\%) & 19 & 18 & 21 \\
\hline Limo (\%) & 24 & 26 & 30 \\
\hline Arcilla (\%) & 50 & 49 & 42 \\
\hline $\mathrm{pH}$ edáfico & 6,0 & 5,8 & 5,8 \\
\hline Materia orgánica (\%) & 6,6 & 7,5 & 9,5 \\
\hline Carbono orgánico (mg g ${ }^{-1}$ de suelo) & 38,1 & 43,5 & 55,2 \\
\hline Nitrógeno total (mg g ${ }^{-1}$ suelo) & 3,1 & 3,7 & 4,3 \\
\hline $\mathrm{C}: \mathrm{N}$ edáfico & 12,3 & 11,8 & 12,8 \\
\hline Fósforo asimilable (mg kg ${ }^{-1}$ ) & 8,4 & 11,8 & 18,9 \\
\hline Calcio asimilable $\left(\mathrm{mg} \mathrm{kg}^{-1}\right)$ & 6.200 & 6.842 & 6.554 \\
\hline Magnesio asimilable (mg kg${ }^{-1}$ ) & 508 & 518 & 517 \\
\hline Potasio asimilable (mg kg-1) & 514 & 499 & 605 \\
\hline
\end{tabular}


La vegetación es característica de la provincia fitogeográfica del espinal, Distrito del Ñandubay. Los bosques fueron seleccionados a base de la representatividad espacial de las unidades de vegetación en toda el área, utilizando una base fisonómico/florística (Braun Blanquet 1979).

Las principales características dasométricas de los bosques se detallan en el cuadro 2, siendo las historias de uso las siguientes:

a) Bosque monoespecífico de A. caven (bosque inicial); proviene de la colonización de ésta especie en un área de cultivo agrícola abandonada desde el año 1998.

b) Bosque secundario dominado por $P$. affinis (bosque secundario) con árboles de hasta 50 años.

c) Bosque maduro dominado por P. nigra y C. tala (bosque maduro) con árboles de la especie dominante (más de 80 años).

Recolección del material foliar. Se seleccionaron cuatro árboles dominantes en cada sitio forestal de las especies A. caven, P. affinis, C. tala y P. nigra cuyos valores de diámetro a la altura $1,3 \mathrm{~m}$ y altura máxima, fueron representativos de la media estimada (cuadro 2).

De cada árbol (con frecuencia mensual) se tomaron muestras de hojas maduras durante el período comprendido entre noviembre del 2008 hasta diciembre de 2010. Para la recolección de las hojas maduras se emplearon tijeras podadoras y las muestras fueron llevadas al laboratorio. Las hojas obtenidas de los cuatro individuos se mezclaron entre sí (León Peláez et al. 2009), previo retirada de ramas y órganos no foliares, luego se secaron en estufa a $70{ }^{\circ} \mathrm{C}$ hasta alcanzar peso constante.

Por otra parte, se recogieron muestras de hojarasca siguiendo la metodología propuesta por Aceñolaza et al. (2009 y 2010); se dispusieron 16 cajas de $0,25 \mathrm{~m}^{2}$ de sección cuadrangular, colocando cuatro cajas bajo copa por especie dominante en cada bosque; se apoyaron sobre el suelo bajo la copa de los árboles a una distancia media de 2 m del tronco (con orientación Este, Oeste, Norte y Sur) por cada individuo seleccionado. Las cajas se colocaron en el campo en Septiembre de 2008 y se comenzaron los muestreos con una frecuencia mensual hasta diciembre de 2010. La hojarasca se clasificó en las siguientes fracciones: a) hojas; b) ramas; c) flores; d) frutos; y d) misceláneas (otro material vegetal o procedente de otras especies ya fueran arbóreas, arbustivas o herbáceas). De las cuatro muestras por especie se extrajo una porción de hojas que se mezclaron (León Peláez et al. 2009), obteniéndose una muestra compuesta de hojas senescentes (Hs) por especie y por mes. Las muestras de hojarasca se secaron en estufa a $70^{\circ} \mathrm{C}$ hasta peso constante.

Tratamiento y análisis químico de las muestras de hojas maduras y hojas senescentes. Todas las muestras se molieron utilizando un molino de bolas (Retsch MM301) y se analizaron elementos totales. Para la determinación del nitrógeno se utilizó un autoanalizador de flujo segmentado (Bran+Luebbe AA3). Para el análisis del resto de los elementos estudiados (Ca, Mg, S y K) se llevó a cabo la calcinación de las muestras en horno de mufla a $500{ }^{\circ} \mathrm{C}$ y posterior ataque ácido; posteriormente se determinaron mediante espectroscopía de emisión atómica usando un plasma de acoplamiento inductivo (ICP-OES). Y, finalmente, el fósforo se determinó siguiendo el método colorimétrico del amarillo de vanado-molibdato de Chapman y Pratt (1979).

Cálculo de índices de retranslocación de nutrientes. Se calcularon dos índices de retranslocación $\left(R c_{1}\right.$ y $\left.R c_{2}\right)$. Ambos índices se determinaron a partir de las diferencias observadas entre las estaciones de primavera y otoño, considerándose las estaciones de máxima y mínima actividad foliar, respectivamente. Se evaluó el proceso de retranslocación considerando solo estas estaciones, a fin de evidenciar las mayores diferencias en cuanto a la concentración de nutrientes en hojas, ya que en general, fueron especies con un patrón de aporte temporal semicaducifolio (Mendoza et al. 2012). Se consideraron las concentraciones de las hojas de los meses correspondientes a cada estación como pseudoréplicas para los cálculos de los índices de retranslocación. Se obtuvieron los valores de $R c_{1}$ y $R c_{2}$ a

Cuadro 2. Valores medios de las principales características dasométricas de las especies dominantes en los bosques inicial (Bi), secundario $(B s)$ y maduro $(B m)$, con sus errores estándares.

Mean values of dasometric characteristics of dominant species in initial (Bi), secondary (Bs), and mature forests (Bm) with their standard errors.

\begin{tabular}{|c|c|c|c|c|c|c|c|}
\hline \multirow{2}{*}{ Bosque } & \multirow{2}{*}{ Especie } & $\mathrm{N}$ & Cob. & DAP & $\mathrm{AB}$ & $\mathrm{D}$ & $\mathrm{H}$ \\
\hline & & (árboles) & $(\%)$ & $(\mathrm{cm})$ & $\left(\mathrm{m}^{2}\right)$ & (árboles ha-1) & $(\mathrm{m})$ \\
\hline$B i$ & A. caven & 541 & $61 \pm 12$ & $16 \pm 5$ & $5,5 \pm 1,2$ & $373 \pm 54$ & $3,4 \pm 0,8$ \\
\hline Bs & P. affinis & 329 & $37 \pm 6$ & $37 \pm 11$ & $8,4 \pm 5,4$ & $227 \pm 48$ & $4,6 \pm 0,9$ \\
\hline \multirow[t]{2}{*}{$\mathrm{Bm}$} & C. tala & 109 & $23 \pm 7$ & $37 \pm 8$ & $2,1 \pm 1,7$ & $75 \pm 35$ & $4,7 \pm 1,7$ \\
\hline & P. nigra & 227 & $47 \pm 12$ & $42 \pm 13$ & $16,1 \pm 4,5$ & $158 \pm 49$ & $6,7 \pm 2,1$ \\
\hline
\end{tabular}

Cobertura en porcentajes de superficie por ha (Cob.); diámetro a la altura del pecho (DAP) en cm; área basal (AB) en $\mathrm{m}^{2}$; densidad (D) en individuos por ha; y altura máxima (h) en $\mathrm{m}$.

Cover in percentage of surface per ha (Cob.); diameter at shoulder height (DAP) in cm; basal area (AB) in $\mathrm{m}^{2}$; density (d) in trees per ha; and height (h) in m. 
partir de las aplicaciones de las ecuaciones 1 y 2, considerando los siguientes pares de meses como se detalla a continuación: octubre - abril/octubre, noviembre - mayo/ noviembre, diciembre - junio/diciembre. En el periodo comprendido entre noviembre del 2008 y diciembre del 2010 , se obtuvieron seis valores para $R c_{1}$ y $R c_{2}$ por especie, los cuales fueron sometidos al correspondiente análisis estadístico. La determinación de los meses a considerar para cada estación, se realizó en función de la caracterización climática para el área de estudio proporcionada por Rojas y Saluso (1987).

El índice $R c_{1}$ se utilizó para determinar la retranslocación considerando las diferencias (Hagen-Thorn et al. 2006) entre la concentración de cada nutriente (N y P, K, $\mathrm{Ca}, \mathrm{Mg}$ y S) en hojas maduras de primavera (recolectadas de la copa de los árboles) y la concentración de cada nutriente en hojas senescentes de otoño (recolectadas de la hojarasca), multiplicado por 100; el valor $R c_{1}$ así obtenido, expresado como porcentaje, permite obtener una aproximación de la cantidad reabsorbida de cada elemento (Del Arco et al. 1991, ecuación 1). Los valores de $R c_{1}$, para cada elemento, permiten indicar las máximas diferencias entre concentraciones de nutrientes en hojas maduras y senescentes, puesto que se considera que en primavera se da la mayor actividad fotosintética (máxima concentración de nutrientes), en contraposición a lo que sucede en otoño:

$R c_{1}(\%)=100 \times \frac{\left(\mathrm{CH}_{1}-\mathrm{CHs}\right)}{\mathrm{CH} m_{1}}$

Donde,

$\mathrm{CHm}_{1}$ = es la concentración de nutriente en hojas maduras de primavera $\mathrm{y}$

$\mathrm{CHs}$ = es la concentración de nutriente en las hojas senescentes recolectadas de la hojarasca en otoño.

Por otra parte $R c_{2}$ se determinó de acuerdo a los valores de la composición química de hojas maduras recolectadas de la copa de los árboles en primavera y de hojas maduras recolectadas de la copa de los árboles en otoño, multiplicado por 100. El valor obtenido de $\mathrm{R} c_{2}$ se expresa también como porcentaje (ecuación 2). Esta adaptación de la ecuación propuesta por Del Arco et al. (1991), como variante de la ecuación 1, permite disminuir los posibles errores en el cálculo de resorción, debido a la posible contaminación de origen edáfico (o por el efecto del pluviolavado) a las que están expuestas las hojas senescentes (que se obtienen de las cajas de recolección de hojarasca apoyadas sobre el suelo, como se describió anteriormente):

$R c_{2}(\%)=100 \times \frac{\left(\mathrm{CHm}_{1}-\mathrm{CHm}_{2}\right)}{\mathrm{CH}_{1}}$

Donde,

$\mathrm{CHm}_{1}$ = es la concentración de nutriente en hojas maduras de primavera y

$\mathrm{CHm}_{2}$ = es la concentración de nutriente en hojas maduras de otoño.
Análisis estadístico. Se compararon los valores de hojas maduras, hojas senescentes y sus razones entre especies (período 2009-2010) mediante un ANDEVA, utilizando un modelo lineal general. El mismo análisis estadístico se realizó para comparar los valores de $R c_{1}$ y $R c_{2}$ entre especies. La homocedasticidad de varianzas se comprobó mediante la prueba de Levene y la normalidad con la prueba de Kolmogorov-Smirnov. En los casos en que el ANDEVA fue significativo se aplicó la prueba de rango múltiple de Tukey $(P<0,05)$ para la comparación de las medias estimadas.

Para todo ello se utilizaron los procedimientos PROC GLM y PROC MIXED del paquete estadístico SAS 9.2 (SAS Institute Inc. 2005).

\section{RESULTADOS}

Concentraciones y abundancias de elementos foliares. Comparando las especies entre sí, se observó un mismo patrón en el orden de importancia en los elementos químicos analizados, tanto en hojas maduras como senescentes. La concentración de nutrientes en hojas maduras siguió la secuencia: nitrógeno $>$ calcio $>$ potasio $>$ fósforo $>$ magnesio > azufre. La excepción fue $C$. tala, donde la concentración de magnesio fue superior a la de fósforo (cuadro 3).

La abundancia de nutrientes en hojas senescentes siguió la misma secuencia que en hojas maduras, a excepción del calcio en A. caven (donde fue el nutriente con mayor concentración) y del magnesio tanto en $A$. caven como en C. tala (sustituyendo éste al fósforo en importancia).

Las concentraciones de nitrógeno en hojas maduras fueron del orden de un $3 \%$ de la masa seca (cuadro 3), siendo C. tala la especie que presentó valores significativamente más altos que el resto. En la concentración de nutriente de las hojas senescentes recolectadas de la hojarasca en otoño se observaron diferencias significativas entre especies, siendo $P$. affinis la que mayor concentración registró (cuadro 3).

En cuanto al fósforo y potasio, las concentraciones en hojas maduras no mostraron diferencias entre especies, mientras que en hojas senescentes el fósforo registró mayor concentración en $P$. affinis y C. tala, y el potasio sólo en C. tala (significativamente diferentes entre especies, en ambos casos).

Tanto las concentraciones de azufre, calcio y de magnesio en las hojas maduras mostraron significativamente valores más altos en C. tala. Por otra parte el azufre en hojas senescentes no mostró diferencias entre especies; pero el calcio y magnesio tuvieron las mayores concentraciones en A. caven y C. tala, respectivamente.

Analizando las razones nitrógeno:fósforo y calcio:potasio en hojas maduras, éstas no tuvieron diferencias significativas entre especies. La razón calcio:magnesio fue máxima en $P$. affinis y mínima en C. tala. En las hojas senescentes la razón nitrógeno:fósforo fue máxima en $A$. caven diferenciándose significativamente de las demás es- 
Cuadro 3. Valores medios de la concentración de nutrientes (N, P, K, Ca, Mg y S) en hojas maduras (Hm) y senescentes (Hs) de las especies dominantes en los bosques inicial (A. caven), secundario (P. affinis) y maduro (C. tala y P. nigra) con errores estándar ( $\mathrm{n}=6$ ).

Mean values of nutrient concentration (N, P, K, Ca, Mg, and S) in mature $(H m)$ and in senescent $(H s)$ leaves of dominant species in initial (A. caven), secondary (P. affinis), and mature (C. Tala and P. nigra) forests, including standard errors $(\mathrm{n}=6)$.

\begin{tabular}{|c|c|c|c|c|c|c|c|c|c|c|}
\hline Especie & & $\mathrm{N}$ & $P$ & S & $\mathrm{Ca}$ & $\mathrm{Mg}$ & $\mathrm{K}$ & $\mathrm{N}: \mathrm{P}$ & Ca:Mg & Ca:K \\
\hline \multicolumn{11}{|c|}{ (mg g ${ }^{-1}$ de materia seca) } \\
\hline \multirow{2}{*}{ A. caven } & $H m$ & $29,2 \pm 0,7 \mathrm{~B}$ & $1,4 \pm 0,1 \mathrm{~A}$ & $0,68 \pm 0,01 B$ & $26,2 \pm 1,6 \mathrm{~A}$ & $1,2 \pm 0,1 \mathrm{~B}$ & $5,9 \pm 0,5 \mathrm{~A}$ & $21,6 \pm 12,6 \mathrm{~A}$ & $21,8 \pm 4,2 \mathrm{~B}$ & $4,4 \pm 1,0 \mathrm{~A}$ \\
\hline & Hs & $24,9 \pm 0,2 d$ & $0,9 \pm 0,1 b$ & $0,59 \pm 0,09 a$ & $25,1 \pm 0,1 \mathrm{a}$ & $1,0 \pm 0,1 b$ & $2,6 \pm 0,1 c$ & $27,6 \pm 1,1 \mathrm{a}$ & $25,1 \pm 7,8 \mathrm{a}$ & $9,6 \pm 2,3 a$ \\
\hline \multirow{2}{*}{ P. affinis } & $\mathrm{Hm}$ & $32,6 \pm 1,5 B$ & $1,4 \pm 0,1 \mathrm{~A}$ & $0,37 \pm 0,02 \mathrm{C}$ & $14,4 \pm 0,7 \mathrm{~B}$ & $0,4 \pm 0,1 \mathrm{C}$ & $3,9 \pm 1,4 \mathrm{~A}$ & $23,4 \pm 7,6 \mathrm{~A}$ & $36,0 \pm 9,3 \mathrm{~A}$ & $3,7 \pm 0,6 \mathrm{~A}$ \\
\hline & Hs & $28,2 \pm 0,2 \mathrm{a}$ & $1,2 \pm 0,1 \mathrm{a}$ & $0,47 \pm 0,12 \mathrm{a}$ & $14,3 \pm 0,1 \mathrm{c}$ & $0,4 \pm 0,1 \mathrm{~d}$ & $3,7 \pm 0,1 b$ & $23,2 \pm 2,0 b$ & $35,7 \pm 10,5 a$ & $3,9 \pm 1,1 b$ \\
\hline \multirow{2}{*}{ C. tala } & $H m$ & $36,4 \pm 0,9 \mathrm{~A}$ & $1,4 \pm 0,1 \mathrm{~A}$ & $0,83 \pm 0,01 \mathrm{~A}$ & $24,5 \pm 1,0 \mathrm{~A}$ & $2,4 \pm 0,2 \mathrm{~A}$ & $5,4 \pm 1,2 \mathrm{~A}$ & $26,5 \pm 7,9 \mathrm{~A}$ & $10,2 \pm 2,1 \mathrm{C}$ & $4,5 \pm 0,9 \mathrm{~A}$ \\
\hline & Hs & $27,1 \pm 0,1 b$ & $1,3 \pm 0,1 \mathrm{a}$ & $0,65 \pm 0,08 a$ & $23,7 \pm 0,1 b$ & $2,4 \pm 0,1 \mathrm{a}$ & $4,9 \pm 0,1 \mathrm{a}$ & $20,2 \pm 1,1 b$ & $9,9 \pm 2,9 b$ & $4,8 \pm 0,8 b$ \\
\hline \multirow{2}{*}{ P. nigra } & $\mathrm{Hm}$ & $31,8 \pm 0,3 \mathrm{~B}$ & $1,3 \pm 0,1 \mathrm{~A}$ & $0,32 \pm 0,02 \mathrm{C}$ & $11,8 \pm 0,9 \mathrm{~B}$ & $0,7 \pm 0,1 \mathrm{C}$ & $4,1 \pm 0,4 \mathrm{~A}$ & $25,2 \pm 12,9 \mathrm{~A}$ & $16,9 \pm 5,2 B$ & $2,9 \pm 1,2 \mathrm{~A}$ \\
\hline & $H s$ & $26,2 \pm 0,2 \mathrm{c}$ & $1,1 \pm 0,1 \mathrm{ab}$ & $0,34 \pm 0,04 a$ & $11,3 \pm 0,1 d$ & $0,6 \pm 0,1 \mathrm{c}$ & $3,7 \pm 0,1 b$ & $23,2 \pm 1,5 b$ & $18,8 \pm 4,1 b$ & $3,1 \pm 1,1 b$ \\
\hline
\end{tabular}

Letras diferentes indican diferencias estadísticamente significativas entre especies, comparando Hm (letras mayúsculas) y Hs (letras minúsculas; prueba de Tukey, $P<0,05$ ).

Different letters mean significant differences among species, comparing $H m$ (upper case) and $H s$ (lower case). Tukey test, $P<0.05$.

pecies; calcio:magnesio fue máxima en A. caven - P. Affnis y mínima en C. tala - P. nigra; y calcio:potasio fue máximo en A. caven y se diferenció estadísticamente de las demás especies.

Comparando las concentraciones de hojas maduras vs hojas senescentes, se observó que el nitrógeno, fósforo, potasio se diferenciaron significativamente entre sí (cuadro 3).

Retranslocación: análisis y comparación de $R c_{1}$ y $R c_{2}$. El porcentaje de retranslocación de nitrógeno fue significativamente diferente entre las familias fabácea y celtidácea, tanto en $R c_{1}$ como en $R c_{2}$. Ambos índices denotaron que la retranslocación en la especie $C$. tala (celtidácea) fue significativamente más alta $(P<0,05)$ que en las demás especies (fabáceas, cuadros 4 y 5 ).

En relación con el fósforo y el potasio, los $R c_{1}$ y $R c_{2}$ mostraron las mismas diferencias significativas entre especies ( $P<0,05$; cuadros 4 y 5 ). En general ambos índices denotan mayor retranslocación de ambos elementos en A. caven. Mientras que el $R c_{1}$ registró valores significativamente mayores en A. caven y menores en las demás especies, sin diferencias significativas entre sí $(P>0,05$; cuadro 4); el $R c_{2}$ evidenció una mayor retranslocación en A. caven y mínimos en $C$. tala, con diferencias estadísticas entre sí $(P<0,05$; cuadro 5$)$.

Los valores de $R c_{1}$ para calcio, magnesio y azufre no mostraron diferencias significativas entre especies $(P>0,05$; cuadro 4). Por el contrario $R c_{2}$ mostró diferencias entre especies $(P<0,05$; cuadro 5$)$, siendo en el azufre y calcio máximos en $A$. caven y mínimos en $C$. tala; y el magnesio máximo en A. caven y P. nigra y mínimo en C. tala (cuadro 5).

\section{DISCUSIÓN}

Concentraciones foliares. En la composición química de las hojas maduras en especies de bosques montanos tropicales (León Peláez et al. 2009) se registró una secuencia de concentración de nutrientes dada por: nitrógeno $>$ potasio $>$ calcio $>$ magnesio $>$ fósforo. En especies de bosques del espinal la secuencia estuvo dada por: nitrógeno $>$ calcio $>$ potasio $>$ fósforo $>$ magnesio $>$ azufre.

Las diferencias observadas denotan la mayor prevalencia de calcio y fósforo en bosques del espinal respecto al potasio y magnesio de bosques montanos tropicales (cuadro 3). Esto podría estar relacionado a una mayor disponibilidad de calcio edáfico en suelos del espinal respecto a los suelos montanos.

En bosques tropicales el contenido de nitrógeno de las hojas maduras osciló entre 13,4 y 17,8 $\mathrm{mg} \mathrm{g}^{-1}$ (Proctor et al. 1983, Veneklaas 1991, León Peláez et al. 2009), mientras que en el espinal pasó a ser casi el doble (entre 29,2 y $36,4 \mathrm{mg} \mathrm{g}^{-1}$; cuadro 3). En el espinal se destacan los altos valores del nitrógeno foliar en hojas maduras (en particular, los de las especies de fabáceas). También los valores del nitrógeno en hojas senescentes (hojas muestreadas en otoño) fueron altos (aunque inferiores a los de hojas maduras), oscilando entre 24,9 y $28,2 \mathrm{mg} \mathrm{g}^{-1}$ ); ya que en esta estación se supone que se ha producido la resorción de elementos móviles como el nitrógeno.

En referencia a los valores de fósforo en hojas maduras registrados en hojas de especies del espinal presentaron un valor medio de 1,3 $\mathrm{mg} \mathrm{g}^{-1}$, superiores a los bosques tropicales (alrededor de 0,9 $\mathrm{mg} \mathrm{g}^{-1}$; Veneklaas 1991, Vera et al. 1999), indicando mejor nutrición. 
Cuadro 4. Valores máximos de retranslocación expresados como porcentajes $\left(\%, R c_{1}\right)$ calculados a partir de los datos de hojas maduras de primavera y hojas senescentes de otoño con errores estándar $(\mathrm{n}=3)$.

Maximum values of resorption expressed as percentages $\left(\%, R c_{1}\right)$ calculated from data from mature leaves in spring and senescent leaves in fall and their standard errors $(\mathrm{n}=3)$.

\begin{tabular}{lcccccc}
\hline Especie & $\mathrm{N}$ & $\mathrm{P}$ & $\mathrm{S}$ & $\mathrm{Ca}$ & $\mathrm{Mg}$ & $\mathrm{K}$ \\
\hline A. caven & $14,5 \pm 2,6 \mathrm{~b}$ & $26,1 \pm 3,7 \mathrm{a}$ & $8,1 \pm 2,4 \mathrm{a}$ & $8,4 \pm 3,0 \mathrm{a}$ & $13,8 \pm 5,8 \mathrm{a}$ & $55,2 \pm 4,6 \mathrm{a}$ \\
P. affinis & $13,3 \pm 3,6 \mathrm{~b}$ & $17,6 \pm 1,3 \mathrm{~b}$ & $7,6 \pm 3,6 \mathrm{a}$ & $5,5 \pm 2,5 \mathrm{a}$ & $4,0 \pm 3,7 \mathrm{a}$ & $12,1 \pm 5,3 \mathrm{~b}$ \\
C. tala & $25,6 \pm 1,7 \mathrm{a}$ & $11,6 \pm 1,7 \mathrm{~b}$ & $13,6 \pm 4,5 \mathrm{a}$ & $4,9 \pm 2,2 \mathrm{a}$ & $7,9 \pm 4,5 \mathrm{a}$ & $22,6 \pm 8,8 \mathrm{~b}$ \\
$P$. nigra & $17,7 \pm 1,4 \mathrm{~b}$ & $13,5 \pm 0,7 \mathrm{~b}$ & $5,7 \pm 3,2 \mathrm{a}$ & $8,7 \pm 3,4 \mathrm{a}$ & $8,0 \pm 4,7 \mathrm{a}$ & $11,3 \pm 4,8 \mathrm{~b}$ \\
\hline
\end{tabular}

Letras diferentes indican diferencias significativas de $R c_{1}$ entre las distintas especies para un mismo elemento (prueba de Tukey, $P<0,05$ ).

Different letters show significant differences in $R c_{1}$ among different species for the same element (Tukey test, $P<0.05$ ).

Cuadro 5. Valores de retranslocación expresados como porcentajes $\left(\%, R c_{2}\right)$ calculados a partir de los datos de hojas maduras de primavera y hojas maduras de otoño con errores estándar $(n=3)$.

Nutrient resorption expressed as percentages $\left(\%, \mathrm{Rc}_{2}\right)$ calculated from data from mature leaves of spring and mature leaves of autumn and their standard errors $(\mathrm{n}=3)$.

\begin{tabular}{lrrrcrc}
\hline \multicolumn{1}{c}{ Especie } & $\mathrm{N}$ & $\mathrm{P}$ & $\mathrm{S}$ & $\mathrm{Ca}$ & $\mathrm{Mg}$ & $\mathrm{K}$ \\
\hline A. caven & $4,4 \pm 2,2 \mathrm{c}$ & $21,3 \pm 2,7 \mathrm{a}$ & $63,7 \pm 5,2 \mathrm{a}$ & $9,8 \pm 1,8 \mathrm{a}$ & $10,2 \pm 1,6 \mathrm{a}$ & $22,7 \pm 3,8 \mathrm{a}$ \\
P. affinis & $8,5 \pm 0,5 \mathrm{~b}$ & $17,6 \pm 2,3 \mathrm{~b}$ & $40,2 \pm 2,6 \mathrm{~b}$ & $7,6 \pm 0,3 \mathrm{~b}$ & $6,4 \pm 2,5 \mathrm{ab}$ & $12,1 \pm 2,5 \mathrm{~b}$ \\
C. tala & $21,1 \pm 2,2 \mathrm{a}$ & $8,9 \pm 2,4 \mathrm{c}$ & $14,6 \pm 3,3 \mathrm{c}$ & $2,4 \pm 0,6 \mathrm{c}$ & $5,8 \pm 0,7 \mathrm{~b}$ & $5,1 \pm 1,4 \mathrm{c}$ \\
$P$. nigra & $9,3 \pm 0,9 \mathrm{~b}$ & $16,4 \pm 1,7 \mathrm{~b}$ & $37,7 \pm 3,8 \mathrm{~b}$ & $6,2 \pm 2,2 \mathrm{~b}$ & $8,6 \pm 1,6 \mathrm{a}$ & $12,1 \pm 2,3 \mathrm{~b}$ \\
\hline
\end{tabular}

Letras diferentes indican diferencias significativas de $R c_{2}$ entre las distintas especies para un mismo elemento (prueba de Tukey, $P<0,05$ ).

Different letters show significant differences in $R c_{2}$ among different species for the same element (Tukey test, $P<0.05$ ).

La relación nitrógeno:fósforo de las hojas maduras del espinal no mostró diferencias significativas entre especies, oscilando entre 21,6 y 26,5. Las hojas senescente de $A$. caven tuvieron un contenido de fósforo significativamente más alto que el del resto de especies (denotando economía en el uso de fósforo en el bosque inicial), que se refleja en los dos índices de retranslocación (cuadros 4 y 5); en contraposición, las especies de fabáceas dominantes en bosques secundarios y bosques maduros no muestran necesidad de economizar fósforo. Los valores de nitrógeno:fósforo foliares del espinal fueron similares a los encontrados por Campo (2007) en bosques tropicales estacionalmente secos de Yucatán (México), aunque son aproximadamente del doble que los valores propuestos por otros autores (Veneklaas 1991, Vera et al. 1999, León Peláez et al. 2009).

Las concentraciones calcio en hojas maduras registradas por León Peláez et al. (2009) fueron del orden de 2,7 y $7,4 \mathrm{mg} \mathrm{g}^{-1}$, mientras que en las de hojas senescentes fueron de 0,4 y 1,2 $\mathrm{mg} \mathrm{g}^{-1}$. En contraposición, el espinal tuvo valores de calcio en hojas maduras que oscilaron entre $11,8 \mathrm{y}$ 26,2 $\mathrm{mg} \mathrm{g}^{-1}$ (siendo máximos en A. caven y C. tala), siendo en hojas senescentes del mismo orden (11,3 y 25,1 $\mathrm{mg} \mathrm{g}^{-1}$ ).

Las concentraciones de magnesio registradas por León Peláez et al. (2009) en hojas maduras se ubicaron en el límite inferior del intervalo definido para un amplio número de bosques tropicales de tierras altas $\left(1,2-3,0 \mathrm{mg} \mathrm{g}^{-1}\right)$, mientras que en hojas senescentes bajó a 0,6-1,0 $\mathrm{mg} \mathrm{g}^{-1}$, debido a pérdidas por lixiviación (Gallardo et al. 1999); valores similares se han encontrado en el espinal.

Consecuentemente, la razón calcio:magnesio en hojas maduras y en hojas senescentes resultaron más altas en el espinal que en bosques tropicales, sugiriendo un desequilibrio en la relación calcio:magnesio, con mayor absorción del calcio en detrimento del magnesio. Los desequilibrios nutricionales en plantas debido a elevadas concentraciones de calcio edáfico fueron estudiados por Xiuming y Papadopoulos (2004) al evaluar desbalances nutricionales en suelos relativos al crecimiento de Solanum lycopersicon L. y Cucumis sativus L. La razón promedio de los cationes de cambio calcio: magnesio edáficas debería ser del orden de 12:1, pero con las condiciones edáficas del espinal (cuadro 1) es probable que las especies estén expuestas a limitaciones en la absorción del magnesio.

Los valores de las concentraciones de potasio en hojas maduras registradas por León Peláez et al. (2009) fueron del orden de 2,7-3,6 $\mathrm{mg} \mathrm{g}^{-1}$, bajando a $0,6-0,8 \mathrm{mg} \mathrm{g}^{-1}$ en las hojas senescentes; estos resultando son inferiores a los valores registrados en especies del espinal (oscilantes entre 3,9-5,9 mg g$^{-1}$ en hojas maduras, que bajan a valores de 2,6-4,9 $\mathrm{mg} \mathrm{g}^{-1}$ ); la fácil lixiviación del potasio foliar explica estas disminuciones en la concentraciones potásicas (Martín et al. 1996).

La razón calcio: potasio denotó escasez de potasio tanto en hojas maduras como en hojas senescentes frente a 
abundancia de calcio, lo cual podría afectar la productividad primaria, ya que el potasio no puede ser reemplazado por otro elemento en sus funciones esenciales del metabolismo (tales como la activación de numerosas enzimas, el control estomático, la fotosíntesis y síntesis de proteínas; Binkley 1993). Estos resultados podrían asimismo estar reflejando el conocido antagonismo entre calcio y magnesio o potasio, según el cual la absorción de iones $\mathrm{Mg}^{2+}$ o $\mathrm{K}^{+}$se ve suprimida por la abundancia de cationes $\mathrm{Ca}^{2+}$ (Binkley 1993).

Análisis de $R c_{1}$ y $R c_{2}$ entre especies. Ambos índices $\left(R c_{1}\right.$ y $R c_{2}$ ) muestran que la retranslocación del nitrógeno en especies de la familia fabácea es significativamente inferior $(P<0,05)$ a la del $C$. tala (familia celtidácea, sin capacidad de fijar biológicamente el nitrógeno atmosférico). Staaf (1982) sugirió que el proceso de resorción es más eficiente en especies con elevados requerimientos nutricionales como el caso de C. tala, posiblemente asociados a la mayor producción de hojas como se pudo registrar en un estudio de aportes de hojarasca en donde se las comparó con especies de fabáceas (Mendoza et al. 2012).

Los bajos valores de la retranslocación para nitrógeno, evidenciados en $R c_{1}$ y $R c_{2}$, en las especies de fabáceas (cuadros 4 y 5) denotan la escasa resorción del nitrógeno. Una elevada concentración de nitrógeno en hojas senescentes supone una baja retranslocación e, indirectamente, un mayor aporte de nitrógeno al suelo con el desfronde (Martín et al. 1996, Gallardo et al. 1999). La menor retranslocación del nitrógeno en especies de fabáceas respecto a lo observado en celtidáceas, está indicando una gran potencialidad de fijar nitrógeno por parte de las primeras, lo cual denota efectivamente la fijación biológica por parte de bacterias simbiontes, e indirectamente implica un mayor retorno de nitrógeno al suelo, siendo esto último de gran valor en los bosques nativos del Espinal Mesopotámico cuyo uso es fundamentalmente silvopastoril (Casermeiro et al. 2001).

En referencia al fósforo y al potasio, la retranslocación fue diferente entre índices ( $R c_{1}$ y $R c_{2}$, cuadros 4 y 5). Mientras que el $R c_{1}$ mostró la mayor retranslocación de fósforo y potasio en $A$. caven respecto a las demás especies comparadas; el $R c_{2}$ denotó diferencias entre Celtidaceae y Fabaceae, siendo mayor la retranslocación del fósforo y potasio en fabáceas. Considerándose el $R c_{2}$ más fiable, es posible que este asociado a una mayor demanda fisiológica de éstos elementos en esta familia.

Respecto a la retranslocación para azufre, calcio y magnesio; el $R c_{1}$ no mostró diferencias significativas entre especies ( $P>0,05$; cuadro 4), en tanto que el $R c_{2}$ (más fiable) parece indicar que hay resorción de estos elementos en fabáceas diferenciándose de la celtidácea que acusó menor resorción. Posiblemente la menor resorción del calcio en C. tala esté asociada a la mayor disponibilidad de este elemento que se acumula en hojas bajo la formación de biominerales de carbonato de calcio (denominados fito- litos, descritos en C. tala por Fernández et al. 2005). En cuanto a la menor resorción del azufre y magnesio podrían atribuirse a las menores demandas nutricionales, a pesar de la mayor producción de hojas como fue observado por Mendoza et al. (2012).

\section{CONCLUSIONES}

- La retranslocación del nitrógeno es significativamente mayor en C. tala (familia Celtidaceae) respecto a lo observado en las especies de la familia Fabaceae; lo cual verificó la hipótesis propuesta.

- El fósforo y el potasio denotan diferencias de la retranslocación entre índices $\left(R c_{1}\right.$ y $\left.R c_{2}\right)$, observándose diferencias entre las fabáceas y la celtidácea solamente en el $R c_{2}$, siendo mayor la resorción en fabáceas, lo que implica mayor demanda nutricional de estos elementos.

- El índice de retranslocación $R c_{2}$ para calcio, azufre y magnesio denota una menor resorción en las fabáceas respecto a la celtidácea, resultando más fiable que el $R c_{2}$ que acusa diferencias significativas entre especies.

- Se concluye que de los índices utilizados para el estudio de la retranslocación foliar en especies dominantes del Espinal Mesopotámico, el $R c_{2}$ resulta tener mayor poder explicativo al maximizar las diferencias observadas entre hojas maduras de primavera y hojas maduras de otoño.

\section{AGRADECIMIENTOS}

Se agradece la financiación parcial otorgada (Proyecto PIDA-2009, U.A.D.E.R.; Paraná, Argentina) y al Programa Erasmus Mundus que posibilitó la estancia en España y el trabajo realizado por el primer autor en la Escuela Técnica Superior de Ingenierías Agrarias (Universidad de Valladolid, España) y en el IRNASa/C.S.I.C (Salamanca, España).

\section{REFERENCIAS}

Aceñolaza PG, LP Zamboni, JF Gallardo Lancho. 2009. Aporte de hojarasca en bosques del predelta del río Paraná (Argentina). Bosque 30(1): 135-145.

Aceñolaza PG, LP Zamboni, EE Rodríguez, JF Gallardo. 2010. Litterfall production in forests located at the Pre-delta area of the Paraná River (Argentina). Annals of Forest Science 67: 311-321.

Binkley D. 1993. Nutrición Forestal, Prácticas de Manejo. México DF, México. Uteha-Limusa. 340 pp.

Braun Blanquet J. 1979. Fitosociología: Bases para el estudio de las comunidades vegetales. Madrid, España. Blume. 820 p.

Campo J. 2007. Estequiometría y acumulación de C en bosques tropicales estacionalmente secos: estudios comparativos y evidencias experimentales. In Gallardo Lancho JF. ed. La Captura de Carbono en Ecosistemas Iberoamericanos. Salamanca, España. Editorial SIFyQA. 85-105 p.

Casermeiro J, AA De Petre, E Spahn, R Valenti. 2001. Efectos del desmonte sobre la vegetación y el suelo. Investigación Agraria: Sistemas y Recursos Forestales 10: 233-244.

Chapman HD y PF Prat. 1979. Métodos de análisis para suelos, 
plantas y aguas. México D.F., México. Trillas. 195 p.

Del Arco JM, A Escudero, GM Vega. 1991. Effects of site characteristics on nitrogen retranslocation from senescing leaves. Ecology 72: 701-708.

Fernández Honaine M, AF Zucol, M Osterriedth. 2005. Biomineralizaciones de sílice en Celtis tala (celtidácea). Boletín $S A B 40$ (3-4): 229-239.

Gallardo JF, A Martín, G Moreno. 1999. Nutrient efficience and resorption in Quercus pyrenaica oak coppices under different rainfall regimes of the 'Sierra de Gata' mountains (Central Western Spain). Annals of Forest Science 56: 321-331.

Hagen-Thorn A, I Varnagiryte, B Nihlgard, K Armolaitis. 2006. Autumn nutrient resorption and losses in four deciduous forest tree species. Forest Ecology and Management 228: 33-39.

INTA (Instituto Nacional de Tecnología Agropecuaria, AR). 2000. Carta de suelos de la República Argentina. Plan Mapa de Suelos de la Provincia de Entre Ríos, Departamento Villaguay. Subsecretaría de Asuntos Agrarios de Entre Ríos, Paraná (Argentina). 130 p.

León Peláez JD, MI González, JF Gallardo. 2009. Retranslocación y eficiencia en el uso de nutrientes en bosques del centro de Antioquia. Revista Colombia Forestal 12: 119-140.

Lewis JP, DE Prado, IM Barberis. 2004. Los remanentes de bosques del Espinal en la provincia de Córdoba. Revista Agromensajes 13: 23-27.

Martín A, I Santa Regina, JF Gallardo.1996. Eficiencia, retranslocación y balance de nutrientes en bosques de Quercus pyrenaica bajo diferente pluviometría en la Sierra de Gata. Ecología 10: 79-93.

Mendoza CA, JF Gallardo, PG Aceñolaza, MB Turrión, V Pando. 2012. Producción de hojarasca de bosques pertenecientes a una secuencia sucesional del Espinal Mesopotámico (R. Argentina). In Gallardo JF. (Coord.), Aguas, suelos y vegetación en cuencas iberoamericanas. Salamanca, España. SiFyQA. p. 177-196.

Muñoz J de D, S Milera, C Romero, A Brizuela. 2005. Bosques nativos y selvas ribereñas en la provincia de Entre Ríos. In Temas de la biodiversidad del litoral fluvial argentino. INSUGEO Miscelánea (Argentina) 14: 169-182.

Proctor J, JM Anderson, SCL Fogden, HW Vallack. 1983. Ecological studies in four contrasting lowland rainforest in Gunung Mulu National Park, Sarawak II. Litterfall, litter standing crop and preliminary observations on herbivory. Journal of Ecology 71: 261-283.

Rojas A, JH Saluso. 1987. Informe Climático de la Provincia de Entre Ríos. INTA EEA Paraná, Publicación Técnica No 14. Paraná (Entre Ríos, Argentina). 25 pp.

SAS Institute Inc. 2005. SAS User’s Guide: Basics, Version 9.1. Cary, N.C., USA.

Staaf H. 1982. Plant nutrient changes in beech leaves during senescence as influenced by site characteristics. Acta Oecologica, Oecologia Plantarum 3: 161-170.

Turrión MB, JF Gallardo, MI González. 2002. Relationships between organic and inorganic $P$ fractions with soil Fe and Al forms in forest soils of the 'Sierra de Gata' mountains (Western Spain). In Violante A, PM Huang, JM Bollag, L Gianfreda eds. Developments in Soil Science. Soil mineralorganic matter-microorganism interactions and ecosystem Health: Dynamics, mobility and transformation of pollutants and nutrients. p: 297- 310.

Veneklaas EJ. 1991. Litterfall and nutrient fluxes in two montane tropical rain forests, Colombia. Journal of Tropical Ecology 7: 319-336.

Vera M, J Cavelier, J Santamaría. 1999. Reabsorción de nitrógeno y fósforo foliar en árboles de bosques montanos en los Andes centrales de Colombia. Revista de Biología Tropical 47: 33-43.

Vitousek PM. 1982. Nutrient cycling and nutrient use efficiency. The American Naturalist 119: 553-572.

Xiuming H, AP Papadopoulos. 2004. Effects of calcium and magnesium on plant growth, biomass partitioning, and fruit yield of winter green house tomato. Horticultural Science 39 (3): 512-515. 
